\title{
Comparative Theoretical Studies of the Reactivity and Stability of Selected Groups of Isomers with Carbon-Oxygen and Carbon-Nitrogen Bonds
}

\author{
Issofa Patouossa*, Eric N. Njabon, Alphonse Emadak, Naomi Zshika \\ Unit of Physical and Theoretical Chemistry of the Laboratory of Applied Physical and Analytical Chemistry, Faculty of Sciences, \\ University of Yaoundé I, Yaoundé I, Cameroon \\ Email: ^patouossa12@yahoo.fr
}

How to cite this paper: Patouossa, I., Njabon, E.N., Emadak, A. and Zshika, N. (2021) Comparative Theoretical Studies of the Reactivity and Stability of Selected Groups of Isomers with Carbon-Oxygen and Carbon-Nitrogen Bonds. Computational Chemistry, 9, 120-130.

https://doi.org/10.4236/cc.2021.92007

Received: March 7, 2021

Accepted: April 27, 2021

Published: April 30, 2021

Copyright $\odot 2021$ by author(s) and Scientific Research Publishing Inc. This work is licensed under the Creative Commons Attribution-NonCommercial International License (CC BY-NC 4.0).

http://creativecommons.org/licenses/by-nc/4.0/

\begin{abstract}
Electronic structure calculations have been carried out to study various closely related isomers with propane backbone which form part of our quantum chemical approach to inter and intra-molecular kinetics. The usefulness of UCA-FUKUI developed by Jesús Sánchez-Márquez to facilitate the theoretical study of chemical reactivity is exploited. All isomers are identified as local minima with single-point calculations on DFT/B3LYP/6-31G(d,p). The increasing order of stability by groups of isomers are group I; propn-2-ol, propan-1-ol, group II; propanone, propanal, group III; Ethylmethanoate, Propanoic acid, Methylethanoate, group IV; N,N-dimethylformamide, propanimino, and propanamide. The trend in reactivity of the various groups of isomers and specific points of nucleophilic and electrophilic attacks are presented. We noticed that most of the properties of these isomers taught at the fundamental levels are proven true theoretically.
\end{abstract}

\section{Keywords}

Electronic Structure, Isomers, Chemical Reactivity, Stability, Nucleophilic and Electrophilic Attacks

\section{Introduction}

Hydrocarbons with three carbon atoms and above have the possibility to form isomers [1]. The two main classes of isomers are constitutional isomers and stereoisomers. Constitutional isomers differ in their structural arrangement of atoms while stereoisomers have different spatial arrangement of atoms. Isomers 
with differences in the structural arrangement of atoms can lead to differences in physical and chemical properties [2]. The carbon-oxygen (C-O) and carbonnitrogen $(\mathrm{C}-\mathrm{N})$ bonds, may lead to structural isomerism by their location in the compound. These bonds are either single, double, or triple bonds as present in functional groups like alcohol, aldehyde, carboxylic acids, amides, and amines, having variable bond lengths and bond energies [3]. Most of the compounds containing $\mathrm{C}-\mathrm{N}$ and $\mathrm{C}-\mathrm{O}$ bonds react in the presence of stronger nucleophiles thus leading to functional group transformations [4]. As the functional groups are transformed, so does the physical and chemical properties. For instance, the energy, stability, and reactivity of these compounds change due to changes in bond types or changes in the arrangement of atoms in the molecule [4] [5].

Aliphatic alcohols with three carbon atoms form two types of constitutional isomers (isopropyl alcohol and propan-1-ol). These alcohols are liquid at room temperature and soluble in water, with secondary alcohols being more basic and primary alcohols being more acidic [6]. Primary alcohols are oxidized to aldehydes and secondary alcohols to ketones [7]. Aldehydes and ketones contain the carbonyl bond which is made up of sigma and pie bond. In this bond, there is greater electron density around the more electronegative oxygen atoms; meanwhile the carbon atom bearing the oxygen atom is more attractive towards nucleophile [5] [6]. The reactivity of carbonyl compounds decreases with increasing chain length. This is due to the increase in the inductive effect of alkyl group which reduces the degree of positive charge on carbon and the steric hindrance about the carbon. Alcohols and carbonyls compounds can be oxidized to carboxylic acids and esters. Carboxylic acid contains both the carbonyl and alcohol functional groups. The electron-withdrawing effect of the oxygen of the carbonyl group polarizes the $\mathrm{OH}$ bond and makes it easier for the hydrogen atom to ionize than in the case of the O-H bond in alcohol [4] [7]. Esters and amides on the other hand are derivatives of carboxylic acid in which the $\mathrm{O}-\mathrm{H}$ group has been replaced with "OR" or $\mathrm{NH}_{2}$ respectively. The reactions are characterized by nucleophilic substitution of the carboxylate and alkoxy or amino group and show similarities to the condensation reactions of aldehydes and ketones. All the substituent groups possess lone pairs of electrons which are conjugated with the carbonyl groups. As the electronegativity of the group increases, the degree of conjugation decreases, and the electron availability about the carbonyl oxygen reduces [8]. The order of electronegativity of this substituent group is as follows: OR' > NH2. However, the overall polarity of the carbonyl group is enhanced by the more electronegative substituents owing to the withdrawing of electrons away from the carbon atom, making it more susceptible to nucleophilic attack [9].

In this work, four families of isomers are studied. The first family is made of propan-1ol and propan-2-ol, the second family consists of propanal and propanone, the third ethyl methanoate, propanoic acid, methyl ethanoate and the fourth is propanamide $\mathrm{N}, \mathrm{N}$-dimethyl formamide and propanimino. A series of theoretical calculations presented herein provided insight into the relative stabil- 
ity of the various isomers. Among the derivatives of carboxylic acid studied in this work, esters are more reactive than amide. Another isomer of the last group call propanimino is partially a derivative of carboxylic acid where the carbonyl group of carboxylic acid is replaced by the $\mathrm{NH}$ group, the properties of this isomer will be studied in subsequent papers. The goal of this article is to complement theoretically, the chemical established principles of these isomers with regards to stability and reactivity towards nucleophilic and electrophilic reagents.

\section{Computational Details}

The geometries of all the molecules as of group I to IV (as shown in Figure 1) were fully optimized using DFT, with functional and basis set B3LYP/6-31G(d,p) as it is in Gaussian 09W package of programs. The calculation of global electrophilicity parameters was done using equations described in the theoretical section. The values electronic properties such as; chemical potential $\mu$, chemical hardness $\eta$, chemical softness, and the energetic properties; such as energy of Homo (EH) or (I), energy of Lumo (EL) or (A), were approximated in terms of the one-electron energies of the HOMO and LUMO frontier molecular orbital respectively using the ground state (GS) of the molecules. The calculation for local reactivity descriptors (fukui function) for the system with; $(\mathrm{N}),(\mathrm{N}+1),(\mathrm{N}$ - 1), electrophilic and nucleophilic fukui electron were performed using, Gaussian $09 \mathrm{~W}$ and UCA-Fukui soft wares. The theoretical details for the method of calculations are shown below.
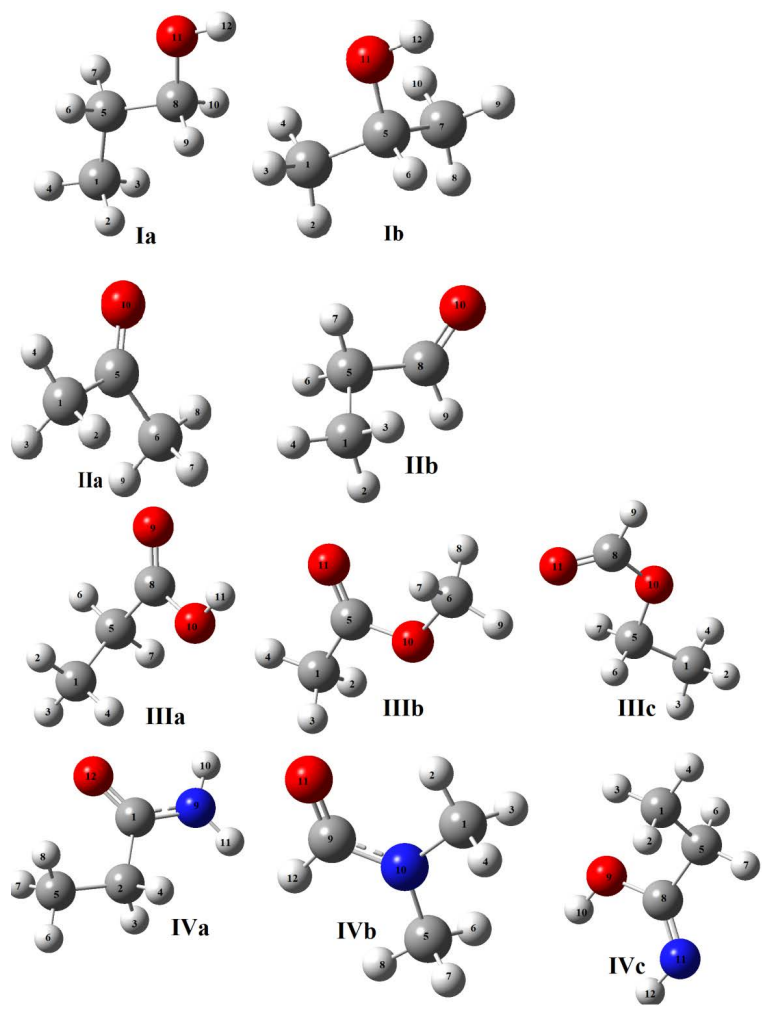

Figure 1. Structures of all sample compounds. 


\subsection{Energetic Parameters}

The thermodynamic stability of a molecule can be predicted theoretically from it electronic energy (energy of optimization), meanwhile experimental stability is predicted base on the enthalpy of formation. To obtain the actual energy of a molecule using DFT, the total electronic energy is added to the zero point vibrational energy. The electronic energy is the total energy content of a molecule and can be obtained through theoretical calculations. Energy of formation is the energy change during a chemical reaction so the. The HOMO and LUMO orbital are the most important orbital in a molecule. These orbital determine how a molecule interacts with other species and give information about reactivity and stability of specific regions of the molecule. According to Koopmans theorem [10], negative of the energy of HOMO (EH) represents the ionization potential (IP) value of the molecule and the negative of the energy of LUMO (EL) represent the Electron Affinity (EA) value

$\mathrm{EH}$ characterized electron-donating ability of a molecule and EL determines the ability of the molecule to accept electrons. Higher values of EH (least negative) indicate better tendency towards donation of electrons and lower values of EL indicate a higher ability to accept electrons [11].

High EH-EL energy gap implies high chemical stability, and small EH-EL means small excitation energy to the manifold of excited state [12].

\subsection{Electronic Parameters}

1) Global reactivity descriptors

The electronic chemical potential $\mu$ is the first derivative of energy with respect to the number of electrons (Equation (1)). It indicates the escape tendency of the electrons

$$
\mu=\left(\frac{\partial E}{\partial V}\right)_{N}
$$

where $E$ is the energy for a system of $N$ elections. The finite difference approximation leads to Equation (2)

$$
\mu=-\frac{1}{2}(I P+E A)
$$

where $I P$ is the ionization potential defined as the difference of the total energy between cationic structure and its neutral species. $E A$ is the election affinity as shown in Equation (4).

$$
\begin{gathered}
I P=E_{\text {cation }}-E_{\text {neutral }} \\
I P=E_{\text {neutral }}-E_{\text {anion }}
\end{gathered}
$$

The hardness, $\eta$, is a global property of the system and measures the resistance to any change in its electron distribution (Equation (5)). The softness can be obtained by taking the inverse of hardness and multiplying by a factor of 0.5 as shown in Equation (6). 


$$
\begin{gathered}
\eta=\frac{1}{2}(I P-E A) \\
S=\frac{1}{2 \eta}
\end{gathered}
$$

The eletrophilicity index $\omega$, which represents the stabilization energy of the molecular system when it is saturated with electrons in the surrounding, can be derived from the chemical potential and hardness as shown in Equation (7).

$$
\omega=\frac{\mu^{2}}{2 \eta}
$$

These Equations (1)-(7), represented the widely accepted global descriptors for reactivity [12] [13] [14] [15].

2) Local reactivity descriptors

Fukui Function (FF) is one of the widely used local density functional descriptors to model chemical reactivity and site selectivity and is defined as the derivative of the electron density $\rho(r)$ with respect to the total number of electrons $N$ in the system, at constant external potential $v(r)$ acting on an electron due to all the nuclei in the system [16] [17].

$$
f(\bar{r})=[\partial \mu / \partial v(\bar{r})]_{N}=[\partial \rho(\bar{r}) / \partial N]_{v(\bar{r})}
$$

The condensed FF is calculated using the procedure proposed by Yang and Mortier, based on a finite difference method

$$
\begin{gathered}
f_{k}^{+}=\left[q_{k}(N+1)-q_{k}(N)\right] \quad \text { for nucleophilic attack } \\
f_{k}^{-}=\left[q_{k}(N)-q_{k}(N-1)\right] \quad \text { for electrophilic attack } \\
f_{k}^{0}=\left[q_{k}(N+1)-q_{k}(N-1)\right] / 2 \text { for free radical attack }
\end{gathered}
$$

$q(N)$ is the charge on kth atom for neutral molecules, $q(N+1)$ and $q(N-1)$ are the same for its anionic and cationic species respectively

\section{Results and Discussion}

In the present work, we have used the entries I, II, III, IV to label the different groups of isomers. The structural/functional group isomers of each group are identified by the notations $a, b, c$. The structures of all the groups of isomers are presented in Figure 1. The energetic properties of the various isomers are presented in table I and II, The electronic properties, global reactivity descriptors and local reactivity descriptors of the sample molecules are presented below in table III and VI respectively.

\subsection{Energetic Properties}

The physical properties like energy and chemical properties like stability (kinectic or thermodynamic stability) of the various isomers gathered from experimental data and computational calculations are summarized in Table 1 below. Table 1 contains the name, dipole moment, total energy, experimental enthalpy 
Table 1. Global reactivity descriptors parameters in electron volt $(\mathrm{eV})$.

\begin{tabular}{|c|c|c|c|c|c|c|}
\hline Group & Molecule & $\begin{array}{l}\text { Dipole moments } \\
\text { in Debye }\end{array}$ & $\begin{array}{l}\text { Energy in } \\
\mathrm{kcal} / \mathrm{mole}\end{array}$ & $\begin{array}{c}\text { Zero point } \\
\text { energy } \\
(\mathrm{kcal} / \mathrm{mole})\end{array}$ & $\begin{array}{l}\text { Total energy in } \\
\mathrm{kcal} / \mathrm{mol}\end{array}$ & $\begin{array}{l}\text { Enthalpy of } \\
\text { formation }\end{array}$ \\
\hline \multirow{2}{*}{ I } & Propan-1-ol & 1.505 & $-121,993.802$ & 67.933 & $-121,925.868$ & -60.970 \\
\hline & Propan-2-ol & 1.578 & $-121,997.516$ & 67.549 & $-121,929.966$ & -65.201 \\
\hline \multirow{2}{*}{ II } & Propanal & 2.761 & -121.233 .401 & 52.717 & $-121,180.684$ & -44.359 \\
\hline & Propanone & 2.768 & $-121,240.566$ & 52.169 & $-168,414.473$ & -51.936 \\
\hline \multirow{3}{*}{ III } & Propanoic acid & 1.681 & $-168,471.292$ & 56.819 & $-168,414.473$ & -108.389 \\
\hline & Ethylmethanoate & 4.218 & $-168,454.383$ & 56.165 & $-168,398.149$ & \\
\hline & Methylethanoate & 4.451 & $-168,454.817$ & 56.234 & $-168,398.652$ & -98.446 \\
\hline \multirow{3}{*}{ IV } & Propanamide & 3.649 & $-155,254.101$ & 63.899 & $-155,190.201$ & -61.879 \\
\hline & $\mathrm{N}, \mathrm{N}$-dimethyl formamide & 3.109 & $-155,988.722$ & 63.959 & $-155,924.763$ & -45.817 \\
\hline & Propanimino & 4.269 & $-155,979.742$ & 64.166 & $-155,915.577$ & \\
\hline
\end{tabular}

of formation, for all the groups of isomers. We evaluated the trend, in the enthalpies of formation obtained through experiment and it ties with the trend in electronic energy obtained through calculation thus our predictions of thermodynamic stability agrees with experimental predictions.

- It is seen from Table 1 that propan-2-ol is thermodynamically more stable with electronic energy-121,929.966 kcal/mol as compare to propan-1-ol with electronic energy $-121,925.868 \mathrm{kcal} / \mathrm{mol}$. Moreover it has been shown through experiments that propan-2-ol is more reactive but less acidic compare to propan-1-ol.

- In the second group of isomers, propanone is the more stable isomer $(-168,414.473 \mathrm{kcal} / \mathrm{mol})$ compare to propanal $(-121,180.684 \mathrm{kcal} / \mathrm{mol})$. In terms of reactivity, propanal is more reactive towards nucleophile than propanone because it is sterically less hindered.

- In the third family of isomers, it evident from their electronic energy that propanoic acid with energy of $-168,414.473 \mathrm{kcal} / \mathrm{mole}$ is more stable compare to methyl ethanoate with electronic energy of $-168,398.652 \mathrm{kcal} / \mathrm{mole}$. In this group, lone pair donation from the hydroxyl oxygen makes the carbonyl oxygen less electrophilic. Under normal reaction conditions, the carboxylic acid wills deprotonate to give the carboxylate which is a very poor electrophile.

- The fourth family constitutes propanamide, N,N-dimethylformamde, and propanimino. N,N-dimethylformamide with electronic energy of $-155,924.763$ $\mathrm{kcal} / \mathrm{mole}$ is the most stable isomer, followed by propanimino with energy of $-155,915.577$ and the least stable is propanamide with energy of $-155,190.201$ $\mathrm{kcal} / \mathrm{mole}$. Nitrogen is less electronegative than oxygen and it is also a better electron pair donor thus amides are less reactive than acid. Electron pair released from nitrogen stabilizes the carbonyl group in amide and decreases the rate of nucleophilic attack. Based on this analysis it is possible that 
$\mathrm{N}, \mathrm{N}$-dimethylformamide is more reactive than propanamide.

The H-L gap is considered excitation energy and determines the ease with which an electron can move from the Lumo to Homo when it energy gap is small. Most hard molecules have large energy gap and soft molecule small energy gap as can be seen in Table 2 below.

Based on the values from Table 2 we can conclude that:

- Propan-1-ol has a smaller Homo-Lumo energy gap thus it is chemically more reactive.

- Propanal is slightly more reactive than Propanone.

- On the other hand, Ethylmethanoate is chemically very stable compared to the other isomers in this group.

- N,N-dimethylformamide is thermodynamically the most stable isomer, it is also the most reactive. The second most reactive in this group is propanamide and the least reactive is Propanimino.

\subsection{Electronic Properties}

Based on our description of electrophilicity, a good electrophile is characterized by high value of $\mu$ and low value of $\eta$. Table 3 below presents the global reactivity descriptor for the various isomers.

It can be deduced from Table 3 that:

- Propan-1-ol is a better electrophile in group one, that is it has a higher propensity to acquire electron from it environment as compared to propan-2-ol. It is also softer.

- Propanal is softer and a better elcetrophile meanwhile Propanone is slightly harder and chemically more stable than Propanal.

- In the 3rd group of isomers, the compound with the greatest propensity to acquire electrons is Methylethanoate followed by Propanoic acid. Ethylmethanoate is chemically stable and hardest isomer in this group.

Table 2. Energies in $\mathrm{eV}$ of the frontier orbital.

\begin{tabular}{ccccc}
\hline Groups of isomers & Names of molecules & $\mathrm{E}_{\text {HOMO }}$ & $\mathrm{E}_{\mathrm{LUMO}}$ & $\Delta \mathrm{E}\left(\mathrm{E}_{\mathrm{H}}-\mathrm{E}_{\mathrm{L}}\right)$ \\
\hline I & Propan-1-ol & -7.495 & 8.387 & 15.880 \\
Propan-2-ol & -9.162 & 8.335 & 17.497 \\
II & Propanal & -7.177 & 0.403 & 7.580 \\
Propanone & -6.876 & 0.731 & 7.607 \\
III & Propanoic acid & -7.016 & 0.416 & 7.432 \\
& Methylethanoate & -6.835 & 0.320 & 7.155 \\
& Ethylmethanoate & -7.155 & 5.654 & 12.809 \\
& Propanamide & -6.080 & 0.980 & 7.060 \\
IV & Propanimino & -6.322 & 0.531 & 6.853 \\
& N,N-dimethyl formamide & -6.645 & 0.761 & 9.406
\end{tabular}


Table 3. Global reactivity descriptors parameters in electron volt $(\mathrm{eV})$.

\begin{tabular}{cccccc}
\hline $\begin{array}{c}\text { Groups of } \\
\text { isomers }\end{array}$ & Names of molecules & $\boldsymbol{\mu}$ & $\boldsymbol{\eta}$ & $\mathrm{S}$ & $\boldsymbol{\omega}$ \\
\hline \multirow{2}{*}{ I } & Propan-1-ol & 0.446 & 15.906 & 46.687 & 0.172 \\
& Propan-2-ol & -0.414 & 17.496 & 42.376 & 0.134 \\
\hline \multirow{2}{*}{ II } & Propanal & -3.388 & 7.581 & 97.681 & 20.590 \\
& Propanone & -3.072 & 7.608 & 97.330 & 16.884 \\
\hline \multirow{2}{*}{ III } & Propanoic acid & -3.301 & 7.431 & 99.624 & 19.931 \\
& Methylethanoate & -3.257 & 7.156 & 103.463 & 20.170 \\
& Ethylmethanoate & -0.751 & 12.810 & 57.797 & 0.599 \\
\hline \multirow{2}{*}{ IV } & Propanamide & -2.550 & 7.061 & 104.867 & 12.527 \\
& N,N-dimethyl formamide & -2.895 & 6.854 & 108.040 & 16.641 \\
\hline
\end{tabular}

- Lastly in the fourth family Propanimino with high chemical stability is the best electrophile in this group of isomers. More so N,N-dimethyl formamde is seen to be chemically very reactive but a poor electrophile with respect to Propanimino and better electrophile than Propanamide.

The Fukui function represents the response of the chemical potential of a system to a change in external potential. As the chemical potential is a measure of the intrinsic acidic or basic strength [14] [18] and local softness incorporates global reactivity, both parameters provide us a pair of indices to demonstrate for example the specific sites of interaction between two reagents. Table 4 below presents the local Fukui function values for the various groups of isomers.

The values in bold are the points for nucleophilic, electrophilic and free radical attacks in the various compounds.

A new index of selectivity toward nucleophilic attack known as Dual Descriptor $\Delta f$ index is discussed elsewhere (Tognetti \& al, 2013); it can be used to characterize an electrophilic attack. It is defined as follow:

$$
\Delta f(r)=\left[f^{+}(r)-f^{-}(r)\right]
$$

If $\Delta f(r)>0$, then the site is favored for a nucleophilic attack, whereas if $\Delta f(r)<$ 0 , then the site could hardly be susceptible to undertake a nucleophilic attack but it may be favored for an electrophilic attack. This is shown in the table above as all unsaturated carbon (carbon bearing the oxygen functional groups) are the points for nucleophilic attack and all the oxygen atoms and some saturated carbon atoms are the point for electrophilic attack.

\section{Conclusion}

The current work reveals that the chemical properties like stability which was predicted based on the trend in electronic energy of the various isomers are in accord with the trend in the enthalpy of formation obtained from experiment. The chemical reactivity of the various isomers, reveals that most isomers which 
Table 4. The local fukui functions data, where O' stands for oxygen with single bond and O” for oxygen with double bonds.

\begin{tabular}{|c|c|c|c|c|c|}
\hline \multicolumn{2}{|c|}{ Compound/N/Z } & $\mathrm{f}^{-}$ & $\mathrm{f}^{+}$ & $\mathrm{f}^{0}$ & $\Delta \mathrm{f}$ \\
\hline \multicolumn{2}{|c|}{ Ia } & & & & \\
\hline $\mathrm{N}$ & Z & & & & \\
\hline 1 & 6 & 0.0001 & 0.0006 & 0.0003 & 0.0004 \\
\hline 5 & 6 & 0.003 & 0.0084 & 0.0043 & 0.0080 \\
\hline 8 & 6 & 0.0116 & 0.5586 & 0.2851 & 0.5470 \\
\hline 11 & 8 & $\underline{0.9878}$ & 0.4317 & $\underline{0.7097}$ & $\underline{-0.5561}$ \\
\hline \multicolumn{2}{|c|}{$\mathrm{I}_{\mathrm{b}}$} & & & & \\
\hline 1 & 6 & 0.0013 & 0.0031 & 0.0022 & 0.0018 \\
\hline 5 & 6 & 0.0240 & $\underline{0.5685}$ & 0.2962 & 0.5445 \\
\hline 7 & 6 & 0.0004 & 0.0021 & 0.0013 & 0.0017 \\
\hline 11 & 8 & $\underline{0.9719}$ & 0.4247 & $\underline{0.6983}$ & $\underline{-0.5473}$ \\
\hline \multicolumn{2}{|c|}{ IIa } & & & & \\
\hline 1 & 6 & 0.0025 & 0.0073 & 0.0049 & 0.0048 \\
\hline 5 & 6 & 0.0156 & 0.6177 & 0.3166 & 0.6021 \\
\hline 6 & 6 & 0.0025 & 0.0073 & 0.0049 & 0.0048 \\
\hline 10 & 8 & 0.9793 & 0.3673 & 0.6733 & $\underline{-0.6119}$ \\
\hline \multicolumn{2}{|c|}{ IIb } & & & & \\
\hline 1 & 6 & 0.0000 & 0.0006 & 0.0003 & 0.0006 \\
\hline 5 & 6 & 0.0011 & 0.0095 & 0.0053 & 0.0085 \\
\hline 8 & 6 & 0.0217 & 0.6088 & 0.3153 & 0.5872 \\
\hline 10 & 8 & 0.9770 & 0.3805 & 0.6788 & $\underline{-0.5964}$ \\
\hline \multicolumn{2}{|c|}{ IIIa } & & & & \\
\hline 1 & 6 & 0.0001 & 0.0010 & 0.0006 & 0.0009 \\
\hline 5 & 6 & 0.0015 & 0.0074 & 0.0044 & 0.0059 \\
\hline 8 & 6 & 0.0222 & 0.6367 & 0.3294 & 0.6145 \\
\hline 9 & 8 & 0.9751 & 0.3464 & 0.6608 & $\underline{-0.6287}$ \\
\hline 10 & 8 & 0.0010 & 0.0078 & 0.0044 & 0.0068 \\
\hline \multicolumn{2}{|c|}{ IIIb } & & & & \\
\hline 1 & 6 & 0.0018 & 0.0074 & 0.0046 & 0.0056 \\
\hline 5 & 6 & 0.0162 & 0.6206 & 0.3184 & 0.6044 \\
\hline 6 & 6 & 0.0001 & 0.0006 & 0.0004 & 0.0005 \\
\hline 10 & 8 & 0.0010 & 0.0061 & 0.0035 & 0.0050 \\
\hline 11 & 8 & 0.9808 & 0.3648 & 0.6728 & $\underline{-0.6160}$ \\
\hline \multicolumn{2}{|c|}{ IIIC } & & & & \\
\hline 1 & 6 & 0.0001 & 0.0015 & 0.0008 & 0.0013 \\
\hline 5 & 6 & 0.0001 & 0.0021 & 0.0011 & 0.0020 \\
\hline 8 & 6 & 0.0172 & 0.5696 & 0.2934 & $\underline{0.5525}$ \\
\hline 10 & 8 & 0.0007 & 0.0035 & 0.0021 & $\overline{0.0029}$ \\
\hline 11 & 8 & 0.9814 & 0.4214 & 0.7014 & -0.5600 \\
\hline \multicolumn{2}{|c|}{ IVa } & & & & \\
\hline 1 & 6 & 0.0146 & 0.6472 & 0.3309 & $\underline{0.6327}$ \\
\hline 2 & 6 & 0.0038 & 0.0006 & 0.0049 & $\overline{0.0022}$ \\
\hline 5 & 6 & 0.0000 & 0.3349 & 0.0003 & 0.0006 \\
\hline 9 & 7 & 0.0029 & 0.0105 & 0.0067 & 0.0076 \\
\hline 12 & 8 & 0.9785 & 0.0060 & 0.6567 & -0.6436 \\
\hline
\end{tabular}




\section{Continued}

\begin{tabular}{|c|c|c|c|c|c|}
\hline \multicolumn{6}{|c|}{ IVb } \\
\hline 1 & 6 & 0.0003 & 0.0000 & 0.0002 & -0.0003 \\
\hline 5 & 6 & 0.0001 & 0.000 & 0.000 & 0.000 \\
\hline 9 & 6 & 0.0214 & 0.6429 & 0.3321 & $\underline{0.6215}$ \\
\hline 10 & 7 & 0.0009 & 0.0132 & 0.0071 & 0.0122 \\
\hline 11 & 8 & 0.9764 & 0.3439 & 0.6602 & -0.6325 \\
\hline \multicolumn{6}{|c|}{ IVc } \\
\hline 1 & 6 & 0.0000 & 0.0012 & 0.0006 & 0.0011 \\
\hline 5 & 6 & 0.0029 & 0.0056 & 0.0042 & 0.0027 \\
\hline 8 & 6 & 0.0087 & 0.5696 & 0.2891 & 0.5609 \\
\hline 9 & 8 & 0.9865 & 0.0057 & 0.4961 & -0.9808 \\
\hline 11 & 7 & 0.0014 & 0.4175 & 0.2095 & 0.4161 \\
\hline
\end{tabular}

are stable thermodynamically are less reactive, except for N,N-dimethylformamide in group IV which is the thermodynamically the most stable and is also the most reactive isomer in this group. Furthermore, most of these isomers react through nucleophilic substitution/addition, thus the isomers are good electrophile else the reaction would not be feasible. However, in the family IV, $\mathrm{N}, \mathrm{N}$-dimethylformamide is a poor electrophile compared to propanamide and propanimino; thus propanimino which is chemically more stable (high energy gap $\triangle \mathrm{EH}-\mathrm{EL}$ ) and has more hardness is the best electrophile in this group. In conclusion this study has confirmed experimental observations on the reactivity, stability, and points of nucleophilic and electrophilic attacks known for this family of compounds.

\section{Conflicts of Interest}

The authors declare no conflicts of interest regarding the publication of this paper.

\section{References}

[1] Kostecki, P., Morrison, R. and Dragun, J. (2005) Hydrocarbons. In: Hillel, D., Ed., Encyclopedia of Soils in the Environment, Academic Press, New York, USA, 217-226. https://doi.org/10.1016/B0-12-348530-4/00098-9

[2] Wang, Z.D., Yang, C., Yang, Z.Y., Brown, C.E., Hollebone, B.P. and Stout, S.A. (2016) 4-Petroleum Biomarker Fingerprinting for Oil Spill Characterization and Source Identification. In: Stout, S.A. and Zhen, D.W., Eds., Standard Handbook Oil Spill Environmental Forensics, 2nd Edition, Academic Press, Cambridge, MA, 131-254. https://doi.org/10.1016/B978-0-12-803832-1.00004-0

[3] Vollhardt, K.P.C. and Schore, E.N. (2007) Organic Chemistry; Structure and Functions. 5th Edition, Freeman and Company, New York.

[4] Francis, A.C. (2000) Functional Group Transformation by Nucleophilic Substitution, Organic Chemistry. 4th Edition, McGraw-Hill Companies, Inc., Rockland, MA, USA.

[5] Haris, C. and Hardcastle, F.D. (2015) Bond Lengths-Valence Relationship for Carbon-Carbon and Carbon-Oxygen Bonds. Journal of the Arkansas Academy of Science, 
69, 45-53.

[6] Clugston, M. and Flemming, R. (2000) Advance Chemistry. Oxford University Press, Oxford.

[7] Raymond, C. (1988) Chemistry. 6th Edition, McGraw-Hill Inc., New York.

[8] Koopmans, T. (1933) Überdie Zuordnung von Wellenfunktionen und Eigenwerten zu den Einzelnen Elektronen Eines Atoms. Physica, 1, 104-113.

https://doi.org/10.1016/S0031-8914(34)90011-2

[9] Sahu, V., Pratibha, S. and Ashok, K. (2014) Impact of Global and Local Reactivity Descriptors on the Hetero-Diels-Alder Reaction of Enaminothione with Various Electrophiles. Journal of the Chilean Chemical Society, 59, 2327-2334. https://doi.org/10.4067/S0717-97072014000100019

[10] Bendjeddou, A., Tahar A.B., Gouasmiab, A. and Villeminc, D. (2016) Quantum Chemical Studies on Molecular Structure and Reactivity Descriptors of some P-NitrophenylTetrathiafulvalenes by Density Functional Theory (DFT). Acta Chimica \& Pharmaceutica Indica, 6, 32-44.

[11] Martinez, J. (2009) Local Reactivity Descriptors from Degenerate Frontier Molecular Orbitals. Chemical Physics Letters, 479, 310-322. https://doi.org/10.1016/j.cplett.2009.07.086

[12] Sánchez-Márquez, J., Zorrilla, D., Sánchez-Coronilla, A., Desireé, M., de los Santos, D.M., Navas, J., Fernández-Lorenzo, C., Alcántara, R. and Martín-Calleja, J. (2014) Introducing "UCA-FUKUI" Software: Reactivity-Index Calculations. Journal of Molecular Modeling, 20, Article No. 2492.

https://doi.org/10.1007/s00894-014-2492-1

[13] Janak, J.F. (1978) Proof That in Density-Functional Theory. Physical Review B, 18, 7165-7168. https://doi.org/10.1103/PhysRevB.18.7165

[14] Meryem, E. and Hasan, T. (2016) Quantum Chemical Studies on the Molecular Structure, Spectroscopic and Electronic Properties of (6-Methoxy-2-oxo-2Hchromen-4-yl)-methyl Pyrrolidine-1-Carbodithioate. Materials Science-Poland, 34, 886-904. https://doi.org/10.1515/msp-2016-0115

[15] Ayers, P.W., Parr, R.G. and Pearson, R.G. (2006) Elucidating the Hard/Soft Acid/Base Principle: A Perspective Based on Half-Reactions. Journal of Chemical Physics, 124, Article ID: 194107. https://doi.org/10.1063/1.2196882

[16] Bendjeddou, A., Abbaz, T., Maache, S., Rehamnia, R., Gouasmia, A.K. and Villemin, D. (2016) Quantum Chemical Descriptors of some Paminophenyltetrathiafulvalenes through Density Functional Theory (DFT). Rasayan Journal of Chemistry, 9, 18-26.

[17] Morell, C., Grand, A. and Toro-Labbe, A. (2005) New Dual Descriptor for Chemical Reactivity. Journal of Chemical Physics A, 109, 205-212. https://doi.org/10.1021/jp046577a

[18] Tognetti, V., Morell, C., Ayers, P.W., Jouberta, L. and Chermetteb, H. (2013) A Proposal for an Extended Dual Descriptor: A Possible Solution When Frontier Molecular Orbital Theory Fails. Physical Chemistry Chimical Physics, 15, Article No. 14465. https://doi.org/10.1039/c3cp51169c 